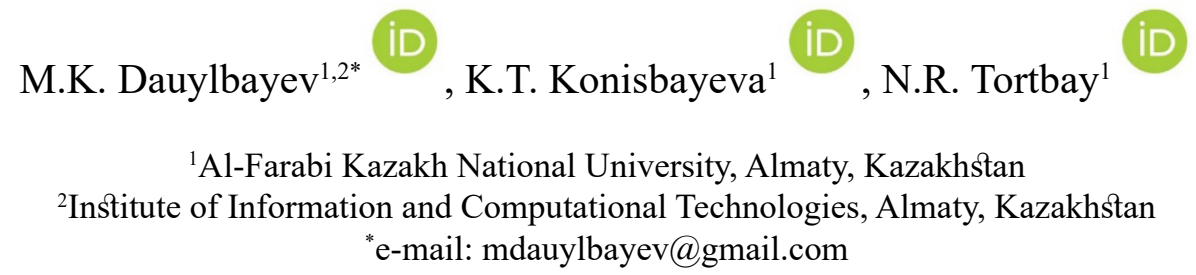

\title{
Integral bvp for singularly perturbed system of differential equations
}

\begin{abstract}
The article presents a two-point integral BVP for singularly perturbed systems of linear ordinary differential equations. The integral BVP for singularly perturbed systems of ordinary differential equations previously has not been considered. The paper shows the influence of nonlocal boundary conditions on the asymptotic of the solution of the regarded BVP and the significant effect of integral terms in the definition of the limiting BVP. An explicit constructive formula for the solution of this BVP using initial and boundary functions of the homogeneous perturbed equation is obtained. A theorem on asymptotic estimates of the solution and its derivatives is given. It is established that the solution of the integral BVP at the point $t=0$ is infinitely large as $\mu \rightarrow 0$.From here, it follows that the solution of the considered boundary value problem has an initial jump of zero order. It is found that the solution of the original integral BVP is not close to the solution of the usual limiting unperturbed BVP. A changed limiting BVP is obtained. The presence of integrals in the boundary conditions leads to the fact that the limiting BVP is determined by the changed boundary conditions. This follows from the presence of the jump and its order. A theorem on the close between the solutions of the original perturbed and changed limiting problems is given.
\end{abstract} BVP.

Key words: singularly perturbation, small parameter, asymptotic, initial jumps, asymptotic estimate,

\section{Introduction}

Many applied problems lead to the consideration of differential systems with small parameters. In the case when the type of the given system changes as small parameters tend to zero, then it is said that it is singularly perturbed. The systematic study of the theory of singularly perturbed equations began with the works of A.N. Tikhonov [1] and V. Vazov [2], where they prove their famous theorems on the passage to the limit in singularly perturbed problems.A significant contribution to the further development of the main directions of the theory was made by L.S. Pontryagin [3], N.N. Bogolyubov, Yu.A. Mitropol'skiy [4], M.I. Vishik, L.A. Lyusternik [5], A.B. Vasilieva, V.F. Butuzov [6], S.A. Lomov [7], Imanaliev M.I. [8] and others.

In the works, [9-12] initial problems with infinitely large valueof an initial data for a sufficiently small value of the parameter were studied. In this case, the solution to the original problem for a sufficiently small value of the parameter approached the solution of the changed degenerate problem. Such initial problems are called Cauchy problems with an initial jump.

BVPs for differential, integro-differential equations with small parameters at the highest derivatives are studied in [13-15]. Here take places initial and boundary jumps phenomena when some derivatives of the solution are unbounded at the left point of the segment or at the both ends. In the work [16] initial problem for piecewise constant argument differential equations is studied.

The boundary value problems considered in [17, $18]$ are local. We consider nonlocal boundary value problems for a system of singularly perturbed differential equations.

For systems of differential equations, such problems have not been considered previously. In these problems, in addition to the initial jumps of the fast and slow variables, the phenomenon of the initial jumps of the integral terms also arises. Thus, the presence of integrals in the boundary conditions leads to a significant modification of the limiting boundary value problem, to which the solution of the original perturbed nonlocal boundary value problem tends. 


\section{Problem statement and auxiliary materials}

We present the following singularly perturbed system of ordinary differential equations of the form

$$
\left\{\begin{array}{l}
\mu z^{\prime \prime}+A_{1}(t) z^{\prime}+B_{1}(t) z+C_{1}(t) y=F_{1}(t) \\
y^{\prime}+A_{2}(t) z^{\prime}+B_{2}(t) z+C_{2}(t) y=F_{2}(t)
\end{array}\right.
$$

with the following boundary conditions

$$
\begin{aligned}
& h_{1} z(t, \mu) \equiv z(0, \mu)=\alpha \\
& h_{2} z(t, \mu) \equiv z(1, \mu)-\int_{0}^{1} \sum_{i=0}^{1} a_{i}(x) z^{(i)}(x, \mu) d x=\beta \\
& h_{3} y(t, \mu) \equiv y(0, \mu)=\gamma
\end{aligned}
$$

where $\mu>0$-small parameter, $\alpha, \beta, \gamma$-given constants, which do not depend on $\mu$.

Now we make two assumptions:

I. $A_{i}(t), B_{i}(t), C_{i}(t), F_{i}(t), i=1,2$ are sufficiently smooth in the segment $0 \leq t \leq 1$;

II. $A_{1}(t) \geq \delta=$ const $>0.0 \leq t \leq 1$

Some other conditions will be imposed later. We view the following homogeneous singularly perturbed differential equation

$$
L_{\mu} z \equiv \mu z^{\prime \prime}+A_{1}(t) z^{\prime}+B_{1}(t) z=0
$$

If the conditions I, II are satisfied, then the fundamental set of solutions $z_{i}(t, \mu), i=1,2$ of the equation (3) has the asymptotic representation as $\mu \rightarrow 0[8]:$

$$
\begin{aligned}
& z_{1}^{(j)}(t, \mu)=z_{10}^{(j)}(t)+O(\mu), j=0,1, \\
& z_{2}^{(j)}(t, \mu)=\frac{1}{\mu^{j}} \exp \left(\frac{1}{\mu} \int_{0}^{t} \kappa(x) d x\right)\left(\kappa^{j}(t) z_{20}(t)+O(\mu)\right), j=0,1,
\end{aligned}
$$

Where $\kappa(t)=-A_{1}(t)<0$, functions $z_{i 0}(t), i=1,2$ are solutions of the problems $A_{1}(t) z^{\prime}{ }_{10}+B_{1}(t) z_{10}=0, z_{10}(0)=1, A_{1}(t) z^{\prime}{ }_{20}+$ $+\left(A_{1}(t)-B_{1}(t)\right) z_{20}=0, z_{10}(0)=1$ respectively have the form

$$
\begin{aligned}
& z_{10}(t)=\exp \left(-\int_{0}^{t} \frac{B_{1}(x)}{A_{1}(x)} d x\right), \\
& z_{20}(t)=\frac{A_{1}(0)}{A_{2}(t)} \exp \left(\int_{0}^{t} \frac{B_{1}(x)}{A_{1}(x)} d x\right)^{\prime}
\end{aligned}
$$

Let the function $K(t, s, \varepsilon)$ as $0 \leq s \leq t \leq 1$ is solution to the problem

$$
\begin{gathered}
L_{\mu} K(t, \mu, \varepsilon)=0, K(s, s, \mu)= \\
=0, K^{\prime}(s, s, \mu)=1
\end{gathered} .
$$

The function $K(t, s, \mu)$ - the Cauchy function, which can be representedas [8]:

$$
K(t, s, \mu)=\frac{W(t, s, \mu)}{W(s, \mu)},
$$

where $W(s, \varepsilon)$ - Wronskian, composed of a fundamental set of solutions $z_{1}(s, \mu), z_{2}(s, \mu)$ equation (3), and $W(t, s, \mu)$ - determinant, obtained from $W(s, \mu)$ by replacing its second row with $z_{1}(s, \mu), z_{2}(s, \mu)$. For the function $K(t, s, \mu)$, the following estimates can be obtained:

$$
\begin{gathered}
K^{(j)}(t, s, \mu)=-\mu \frac{z_{10}^{(j)}(t)}{z_{10}(s) \kappa(s)}+ \\
+\mu^{1-j} \exp \left(\frac{1}{\mu} \int_{s}^{t} \kappa(x) d x\right) \frac{\kappa^{j}(t) z_{20}(t)}{z_{20}(s) \kappa(s)}+ \\
+O\left(\mu^{2}+\mu^{2-j} \exp \left(\frac{1}{\mu} \int_{s}^{t} \kappa(x) d x\right)\right), j=0,1,
\end{gathered}
$$

where $z_{i 0}(t), i=1,2$ is expressed by formula (5). 
Now we introduce the boundary functions of the function $\Phi_{i}(t, \mu), i=1,2$, which are solutions of the following problem

$$
L_{\mu} \Phi_{i}(t, \mu)=0, h_{k} \Phi_{i}(t, \mu)=\delta_{k i}, k, i=1,2,
$$

where $\delta_{k i}-$ Kronecker symbol. Consider the determinant

$$
\Delta(\mu)=\left|\begin{array}{ll}
h_{1} z_{1}(t, \mu) & h_{1} z_{2}(t, \mu) \\
h_{2} z_{2}(t, \mu) & h_{2} z_{2}(t, \mu)
\end{array}\right|
$$

For the determinant $\Delta(\mu)$, taking into account (2), (4), (5), the asymptotic representation as $\mu \rightarrow 0$ is valid

$$
\Delta(\mu)=\Delta_{0}+O(\mu)
$$

where $\Delta_{0}=a_{1}(0)-h_{2} z_{10}(t)$.

III. Let $\Delta_{0} \neq 0$

Boundary functions $\Phi_{i}(t, \mu), i=1,2$ can be represented in the form [8]:

$$
\Phi_{i}(t, \mu)=\frac{\Delta_{i}(t, \mu)}{\Delta(\mu)}, i=1,2
$$

where $\Delta_{i}(t, \mu)$ is the determinant obtained by replacing the $i$-th row with the fundamental set of solutions $z_{1}(s, \mu), z_{2}(s, \mu)$ to equation (3).

For the boundary functions $\Phi_{i}(t, \mu), i=1,2$ from (11), with considering (4), (10), one can obtain the following asymptotic representations as $\mu \rightarrow 0$ :

$$
\begin{aligned}
& \Phi_{1}^{(j)}(t, \mu)= \frac{a_{1}(0) z_{10}^{(j)}(t)}{\Delta_{0}}-\frac{1}{\mu^{j}} \exp \left(\frac{1}{\mu} \int_{0}^{t} \kappa(x) d x\right) \frac{\kappa^{j}(t) z_{20}(t) z_{10}(t)}{\Delta_{0}}+ \\
&+O\left(\mu+\frac{1}{\mu^{j-1}} \exp \left(\frac{1}{\mu} \int_{0}^{t} \kappa(x) d x\right)\right), j=0,1, \\
& \Phi_{2}^{(j)}(t, \mu)=-\frac{z_{10}^{(j)}(t)}{\Delta_{0}}+\frac{1}{\mu_{j}} \exp \left(\frac{1}{\mu} \int_{0}^{t} \kappa(x) d x\right) \frac{\kappa^{j}(t) z_{20}(t)}{\Delta_{0}}+ \\
&+O\left(\mu+\frac{1}{\mu^{j-1}} \exp \left(\frac{1}{\mu} \int_{0}^{t} \kappa(x) d x\right)\right), j=0,1,
\end{aligned}
$$

\section{Main results.}

From the system (1), we find

$$
\begin{gathered}
y(t, \mu)=\gamma e^{-\int_{0}^{t} C_{2}(s) d s}+\int_{0}^{t}\left(F_{2}(s)-\right. \\
\left.-A_{2}(s) z^{\prime}(s, \mu)-B_{2}(s) z(s, \mu)\right) e^{-\int_{s}^{t} C_{2}(p) d p} d s
\end{gathered}
$$

Let us substitute in the first equation of system (1) the expression (13) with respect to $z(s, \mu)$,we acquire the Volterra integro differential equation

$$
\begin{gathered}
L_{\mu} y \equiv \mu z^{\prime \prime}+A_{1}(t) z^{\prime}+B_{1}(t) z= \\
=F(t)+\int_{0}^{1} \sum_{i=0}^{1} H_{i}(t, s) z^{(i)}(s, \mu) d s
\end{gathered}
$$

with the following boundary conditions

$$
\begin{aligned}
& h_{1} z(t, \mu) \equiv z(0, \mu)=\alpha \\
& h_{2} z(t, \mu) \equiv z(1, \mu)-\int_{0}^{1} \sum_{i=0}^{1} a_{i}(x) z^{(i)}(x, \mu) d x=\beta
\end{aligned}
$$

where 


$$
\begin{gathered}
F(t)=F_{1}(t)-\gamma C_{1}(t) e^{-\int_{0}^{t} C_{2}(s) d s}-\int_{0}^{t} C_{1}(t) F_{2}(s) e^{-\int_{s}^{t} C_{2}(p) d p} d s, \\
H_{0}(t, s)=C_{1}(t) B_{2}(s) e^{-\int^{t} C_{2}(p) d p}, H_{1}(t, s)=C_{1}(t) A_{2}(s) e^{-\int_{s}^{t} C_{2}(p) d p},
\end{gathered}
$$

We seek the solution to the BVP (14), (15) in the where form:

$$
\begin{gathered}
z(t, \mu)=C_{1} \Phi_{1}(t, \mu)+C_{2} \Phi_{2}(t, \mu)+ \\
+\frac{1}{\mu} \int_{0}^{t} K(t, s, \mu) u(s, \mu) d s
\end{gathered}
$$

where $C_{i}, i=1,2 \quad$ - unknown constants, $u(t, \mu)$ satisfies the integral equations

$$
u(t, \mu)=f(t, \mu)+\int_{0}^{t} H(t, s, \mu) u(s, \mu) d s .
$$

Here

$$
\begin{aligned}
f(t, \mu) & =F(t)+C_{1} \int_{0}^{t} \sum_{i=0}^{1} H_{i}(t, s) \Phi_{1}^{(j)}(s, \mu) d s+ \\
& +C_{2} \int_{0}^{t} \sum_{i=0}^{1} H_{i}(t, s) \Phi_{2}^{(j)}(s, \mu) d s,
\end{aligned}
$$

$H(t, s, \mu)=\frac{1}{\mu} \int_{s}^{t} \sum_{i=0}^{1} H_{i}(t, p) K^{(j)}(p, s, \mu) d p$

The kernel $H(t, s, \mu)$ is continuous in the domain $0 \leq t \leq 1,0 \leq s \leq t$ and is bounded for sufficiently small $\mu$. Therefore, the resolvent $R(t, s, \mu)$ of the kernel $H(t, s, \mu)$ is also limited andalso has the following asymptotic representation

$$
R(t, s, \mu)=\bar{R}(t, s)+O(\mu)
$$

where $\bar{R}(t, s)$ is the part of the resolvent $R(t, s, \mu)$ which do not depend on $\mu$.

Solving equation (18) using the resolvent we find

$$
z(t, \mu)=\sum_{i=1}^{2} C_{i} Q_{i}(t, \mu)+P(t, \mu),
$$

$$
\begin{gathered}
Q_{i}(t, \mu)=\Phi_{i}(t, \mu)+\frac{1}{\mu} \int_{o}^{t} K(t, s, \mu) \bar{\phi}_{i}(s, \mu) d s \\
i=1,2, P(t, \mu)=\frac{1}{\mu} \int_{0}^{t} K(t, s, \mu) \bar{F}(s, \mu) d s \\
\bar{\phi}_{i}(t, \mu)=\int_{0}^{t} \sum_{j=0}^{1} \bar{H}_{j}(t, s, \mu) \Phi_{i}^{(j)}(s, \mu) d s, \\
i=1,2, \quad \bar{F}(t, \mu)=F(t)+\int_{0}^{t} R(t, s, \mu) F(s) d s, \\
\bar{H}(t, s, \mu)=H_{j}(t, s)+\int_{s}^{t} R(t, p, \mu) H_{i}(p, s) d s, \\
j=0,1
\end{gathered}
$$

For the function $\bar{\varphi}_{i}(t, \mu), \bar{F}(t, \mu), \bar{H}_{j}(t, s, \mu)$ from (23), in view of (20), (12), we get the following asymptotic representations as $\mu \rightarrow 0$ :

$$
\begin{gathered}
\bar{\phi}_{1}(t, \mu)=\bar{\phi}_{1}(t)-\bar{H}_{1}(t, t) \frac{z_{10}(t) z_{20}(t)}{\Delta_{0}} e^{\frac{1}{\mu} \int_{0}^{t} \kappa(x) d x}+{ }_{(24)} \\
+O(\mu), \bar{F}(t, \mu)=\bar{F}(t)+O(\mu) \\
\bar{\phi}_{2}(t, \mu)=\bar{\phi}_{2}(t)-\bar{H}_{1}(t, t) \frac{z_{20}(t)}{\Delta_{0}} e^{\frac{1}{\mu} \int_{0}^{t} \kappa(x) d x}+ \\
+O(\mu), \bar{H}_{j}(t, s, \mu)=\bar{H}_{j}(t, s)+O(\mu), j=0,1
\end{gathered}
$$

where

$$
\begin{gathered}
\bar{\varphi}_{1}(t)=\bar{H}_{1}(t, 0) \frac{h_{2} z_{10}(t)}{\Delta_{0}}+\int_{0}^{t} \sum_{i=0}^{1} \bar{H}_{i}(t, s) \frac{a_{1}(0) z_{10}^{(i)}(s)}{\Delta_{0}} d s, \\
\bar{\varphi}_{2}(t)=-\frac{\bar{H}_{1}(t, 0)}{\Delta_{0}}-\int_{0}^{t} \sum_{i=0}^{1} \bar{H}_{i}(t, s) \frac{z_{10}^{(i)}(s)}{\Delta_{0}} d s,
\end{gathered}
$$

Now, from (22), in consideration of (8), (12), (24) we derivethe asymptotic representations: 


$$
\begin{aligned}
& Q_{1}^{(j)}(t, \mu)=\frac{a_{1}(0) z_{10}^{(j)}(t)}{\Delta_{0}}-\int_{0}^{t} \frac{z_{10}^{(j)}(t) \bar{\phi}_{1}(s)}{z_{10}(s) \kappa(s)} d s-\frac{\kappa^{j-2}(t) \bar{\phi}_{1}(t)}{\mu^{j-1}}- \\
& -\frac{1}{\mu} e^{\frac{1}{\mu} \int_{0}^{t} \kappa(x) d x} \frac{\kappa^{j}(t) z_{20}(t) h_{2} z_{10}(t)}{\Delta_{0}}\left(1+\int_{0}^{t} \frac{\bar{H}_{1}(s, s)}{\kappa(s)} d s\right)+O\left(\mu+\frac{1}{\mu^{j-1}} e^{\frac{1}{\mu} \int_{0}^{t} \kappa(x) d x}\right), \\
& Q_{2}^{(j)}(t, \mu)=-\frac{z_{10}^{(j)}(t)}{\Delta_{0}}-\int_{0}^{t} \frac{z_{10}^{(j)}(t) \bar{\phi}_{2}(s)}{z_{10}(s) \kappa(s)} d s-\frac{\kappa^{j-2}(t) \bar{\phi}_{2}(t)}{\mu^{j-1}}+ \\
& +\frac{1}{\mu} e^{\frac{1}{\mu} \int_{0}^{t} \kappa(x) d x} \frac{\kappa^{j}(t) z_{20}(t)}{\Delta_{0}}\left(1+\int_{0}^{t} \frac{\bar{H}_{1}(s, s)}{\kappa(s)} d s\right)+O\left(\mu+\frac{1}{\mu^{j-1}} e^{\frac{1}{\mu} \int_{0}^{t} \kappa(x) d x}\right), \\
& P^{(j)}(t, \mu)=-\int_{0}^{t} \frac{z_{10}^{(j)}(t) \bar{F}(s)}{z_{10}(s) \kappa(s)} d s-\frac{\kappa^{j-2}(t) \bar{F}(t)}{\mu^{j-1}}- \\
& -\frac{1}{\mu^{j-1}} e^{\frac{1}{\mu} \int_{0}^{t} \kappa(x) d x} \frac{\kappa^{j}(t) z_{20}(t) \bar{F}(0)}{\kappa^{2}(0)}+O\left(\mu+\frac{1}{\mu^{j-2}} e^{\frac{1}{\mu} \int_{0}^{t} \kappa(x) d x}\right),
\end{aligned}
$$

From (21), in consideration of (15), we determine the unknown constants $C_{i}, i=1,2$ from the system

$$
\left\{\begin{array}{l}
C_{1} h_{1} Q_{1}(t, \mu)+C_{2} h_{1} Q_{2}(t, \mu)=\alpha-h_{1} P(t, \mu), \\
C_{1} h_{2} Q_{1}(t, \mu)+C_{2} h_{2} Q_{2}(t, \mu)=\beta-h_{2} P(t, \mu)
\end{array}\right.
$$

where the asymptotic representations are valid

$$
\begin{aligned}
& h_{1} Q_{1}(t, \mu)=1, \quad h_{1} Q_{2}(t, \mu)=0, \quad h_{1} P(t, \mu)=0 \\
& h_{2} Q_{1}(t, \mu)=-\int_{0}^{1} \frac{\bar{z}_{10}(s) \bar{\phi}_{1}(s)}{z_{10}(s) \kappa(s)} d s+O(\mu), \\
& h_{2} Q_{2}(t, \mu)=1-\int_{0}^{1} \frac{\bar{z}_{10}(s) \bar{\phi}_{2}(s)}{z_{10}(s) \kappa(s)} d s+O(\mu) \\
& h_{2} P(t, \mu)=-\int_{0}^{1} \frac{\bar{z}_{10}(s) \bar{F}(s)}{z_{10}(s) \kappa(s)} d s+O(\mu), \\
& \bar{z}_{10}(t)=z_{10}(1)-a_{1}(s) z_{10}(s)-\int_{s}^{1} \sum_{i=0}^{1} a_{i}(x) z_{10}^{(i)}(x) d x
\end{aligned}
$$

Let the condition be satisfied

$$
\text { IV. } \omega_{0}=1-\int_{0}^{1} \frac{\bar{z}_{10}(\mathrm{~s}) \bar{\varphi}_{2}(s)}{z_{10}(s) \mu(s)} d s \neq 0
$$

Then from system (27), in view of (28), we have

where

$$
C_{1}=\alpha, C_{2}=\omega+O(\mu),
$$

$$
\omega=\frac{1}{\omega_{0}}\left(\beta+\int_{0}^{1} \frac{\bar{z}_{10}(s)\left(\alpha \bar{\varphi}_{1}(s)+\bar{F}(s)\right)}{z_{10}(s) \kappa(s)} d s\right)
$$

Theorem 1. Underconditions I-IV there exists a positive constant $\mu_{0}$ that for $\mu \in\left(0, \mu_{0}\right]$ there exists a unique solution of problem (1), (2) which satisfies the following asymptotic estimates as $\mu \rightarrow 0$ :

$$
\begin{gathered}
\left|z^{(j)}(t, \mu)\right| \leq C\left(\left|\alpha a_{1}(0)-\beta\right|+\left|\alpha A_{2}(0)+\gamma\right|+\right. \\
\left.+\max _{0 \leq t \leq 1}\left|F_{1}(t)\right|+\max _{0 \leq t \leq 1}\left|F_{2}(t)\right|\right)\left(1+\frac{1}{\mu^{j}} e^{-\delta \frac{t}{\mu}}\right) \\
|y(t, \mu)| \leq C\left(\left|\alpha a_{1}(0)-\beta\right|+\mid \alpha A_{2}(0)+\right. \\
\left.+\gamma\left|+\max _{0 \leq t \leq 1}\right| F_{1}(t)\left|+\max _{0 \leq t \leq 1}\right| F_{2}(t) \mid\right)\left(1+e^{-\delta \frac{t}{\mu}}\right)
\end{gathered}
$$

where $C>0, \delta>0$-some constants independent of $\mu$.

Proof. In view of (26), (29) from (21) for solutions of the problem (1), (2) we derive the following asymptotic representations as $\mu \rightarrow 0$ : 


$$
\begin{aligned}
& z^{(j)}(t, \mu)=\frac{\left(\alpha a_{1}(0)-\omega\right) z_{1}^{(j)}(t)}{\Delta_{0}}-\int_{0}^{t} \frac{z_{10}^{(j)}(t)\left(\alpha \bar{\phi}_{1}(s)+\omega \bar{\phi}_{2}(s)+\bar{F}(s)\right)}{z_{10}(s) \kappa(s)} d s- \\
& \frac{\kappa_{j-2}(t)\left(\alpha \bar{\phi}_{1}(s)+\omega \bar{\phi}_{2}(s)+\bar{F}(s)\right)}{\mu^{j-1}}-\frac{\kappa^{j}(t) z_{20}(t)\left(\alpha h_{2} z_{10}(t)+\omega\right)}{\mu^{j} \Delta_{0}}\left(1+\int_{0}^{t} \frac{\bar{H}_{1}(s, s)}{\kappa(s)} d s\right)+ \\
& +O\left(\mu+\frac{1}{\mu^{j-1}} e^{\frac{1}{\mu} \int_{0}^{t} \kappa(x) d x}\right), j=0,1 \\
& y(t, \mu)=\left(\gamma-\frac{A_{2}(0)\left(\alpha h_{2} z_{10}(t)+\omega\right)}{\Delta_{0}}\right) e^{-\int_{0}^{t} C_{2}(s) d s}+\int_{0}^{t}\left\{F_{2}(s)-A_{2}(s)\left[\frac{\left(\alpha a_{1}(0)-\omega\right) z^{\prime}{ }_{10}(s)}{\Delta_{0}}-\right.\right. \\
& \left.\int_{0}^{s} \frac{z_{10}^{\prime}(s)\left(\alpha \bar{\phi}_{1}(p)+\omega \bar{\phi}_{2}(p)+\bar{F}(p)\right)}{z_{10}(p) \kappa(p)} d p-\frac{\alpha \bar{\phi}_{1}(s)+\omega \bar{\phi}_{2}(s)+\bar{F}(s)}{\kappa(s)}\right]- \\
& \left.-B_{2}(s)\left[\frac{\left(\alpha a_{1}(0)-\omega\right) z_{10}(s)}{\Delta_{0}}-\int_{0}^{s} \frac{z_{10}(s)\left(\alpha \bar{\phi}_{1}(p)+\omega \bar{\phi}_{2}(p)+\bar{F}(p)\right)}{z_{10}(p) \kappa(p)} d p\right]\right\} e^{\int^{-s} c_{2}(p) d p} d s+ \\
& +\frac{A_{2}(t) z_{20}(t)\left(\alpha h_{2} z_{10}(t)-\omega\right)}{\Delta_{0}}\left(1+\int_{0}^{t} \frac{\bar{H}_{1}(s, s)}{\kappa(s)} d s\right) e^{\frac{1}{\mu} \int_{0}^{t} \kappa(x) d x}+O(\mu)
\end{aligned}
$$

We transform in (32) the expressions $\alpha a_{1}(0)-\omega, \gamma-\frac{\left.A_{2}(0)\left(\alpha h_{2} z_{10}(t)-\omega\right)\right)}{\Delta_{0}}$ to the form

$$
\begin{gathered}
\alpha a_{1}(0)-\omega=\frac{1}{\omega_{0}}\left(\alpha a_{1}(0)-\beta-\int_{0}^{1} \frac{z_{10}(s)\left(\alpha \bar{\phi}_{1}(s)+\alpha a_{1}(0) \bar{\phi}_{2}(s)+\bar{F}(s)\right)}{z_{10}(s) \kappa(s)} d s\right) \\
\gamma-\frac{A_{2}\left(\alpha h_{2} z_{10}(t)-\omega\right)}{\Delta_{0}}=\gamma+\alpha A_{2}(0)-\frac{A_{2}(0)}{\Delta_{0} \omega_{0}}\left(\alpha a_{1}(0)-\beta-\int_{0}^{s} \frac{\bar{z}_{10}(s)\left(\bar{F}(s)-\alpha \bar{H}_{1}(s, 0)\right)}{z_{10}(s) \kappa(s)} d s\right)
\end{gathered}
$$

and the expressions $\alpha \bar{\varphi}_{1}(t)+\omega \bar{\varphi}_{2}(t)+\bar{F}(t), \alpha \bar{\varphi}_{1}(t)+\alpha a_{1}(0) \bar{\varphi}_{2}(t)+\bar{F}(t), \bar{F}(t)-\alpha \bar{H}_{1}(t, 0)$ to the form

$$
\begin{gathered}
\alpha \bar{\varphi}_{1}(t)+\omega \bar{\varphi}_{2}(t)+\bar{F}(t)=\frac{1}{\omega_{0}}\left(\alpha \bar{\varphi}_{1}(t)+\beta \bar{\varphi}_{2}(t)+\bar{F}(t)\right)= \\
=\frac{1}{\omega_{0}}\left(\frac{\alpha a_{1}(0)-\beta}{\Delta_{0}} \bar{H}_{1}(t, 0)+\frac{\alpha a_{1}(0)-\beta}{\Delta_{0}} \int_{0}^{s} \sum_{i=0}^{1} \bar{H}_{i}(t, s) z_{10}^{(i)}(s) d s+\bar{F}(t)-\alpha \bar{H}_{1}(t, 0)\right) \\
\alpha \bar{\varphi}_{1}(t)+\alpha a_{1}(0) \bar{\varphi}_{2}(t)+\bar{F}(t)=\bar{F}(t)-\alpha \bar{H}_{1}(t, 0),
\end{gathered}
$$




$$
\begin{gathered}
\bar{F}(t)-\alpha \bar{H}_{1}(t, 0)=-\left(\alpha A_{2}(0)+\gamma\right)\left(C_{1}(t) e^{-\int_{0}^{t} C_{2}(p) d p}+\int_{0}^{t} \bar{R}(t, s) C_{1}(s) e^{-\int_{0}^{s} C_{2}(p) d p} d s\right)+ \\
+F_{1}(t)+\int_{0}^{t} \bar{R}(t, s) F_{1}(s) d s-\int_{0}^{t}\left(C_{1}(t)+\int_{s}^{t} \bar{R}(t, p) C_{1}(p) d p\right) F_{2}(s) e^{-\int_{s}^{t} C_{2}(p) d p} d s
\end{gathered}
$$

Then from (34) the asymptotic estimate will be represented in the form

$$
\begin{gathered}
\left|\alpha \bar{\varphi}_{1}(t)+\omega \bar{\varphi}_{2}(t)+\bar{F}(t)\right| \leq \\
\leq C\left(\left|\alpha a_{1}(0)-\beta\right|+\left|\alpha A_{2}(0)+\gamma\right|+,\right. \\
\left.+\max _{0 \leq t \leq 1}\left|F_{1}(t)\right|+\max _{0 \leq t \leq 1}\left|F_{2}(t)\right|\right) \\
\left|\alpha \bar{\varphi}_{1}(t)+\alpha a_{1}(0) \bar{\varphi}_{2}(t)+\bar{F}(t)\right| \leq \\
\leq C\left(\left|\alpha A_{2}(0)+\gamma\right|+\max _{0 \leq t \leq 1}\left|F_{1}(t)\right|+\max _{0 \leq t \leq 1}\left|F_{2}(t)\right|\right)
\end{gathered}
$$

Now, from asymptotic formulas (32), in view of (33) - (35), we get estimates (31). Theorem 1 is proved.

Theorem 1 implies that

$$
\begin{gathered}
z(0, \mu)=O(1), z^{\prime}(0, \varepsilon)=O\left(\frac{1}{\mu}\right), \\
y(0, \mu)=O(1), y^{\prime}(0, \mu)=O\left(\frac{1}{\mu}\right), \mu \rightarrow 0
\end{gathered}
$$

Consequently, the solution of the integral BVP (1), (2) has an initial jump of zero order at the left point of the segment.

\section{A changed unperturbed problem.}

In view of the problem (1), (2) as $\mu=0$ we obtain the following BVP

$$
\left\{\begin{array}{c}
A_{1}(t) \widetilde{z}^{\prime}+B_{1}(t) \widetilde{z}+C_{1}(t) \tilde{y}=F_{1}(t) \\
\tilde{y}^{\prime}+A_{2}(t) \widetilde{z}^{\prime}+B_{2}(t) \widetilde{z}+C_{2}(t) \tilde{y}=F_{2}(t) \\
h_{1} \tilde{z}(t)=\alpha, h_{3} \tilde{y}(t)=\gamma
\end{array}\right.
$$

Now, we investigate the limit passage between the solutions of the perturbed problem (1), (2) and the usual unperturbed problem (37), (38). In system (1), (2) we carry out a change of variables by the formulae $u(t, \mu)=z(t, \mu)-\widetilde{z}(t), v(t, \mu)=y(t, \mu)-\widetilde{y}(t)$.

Then we get the system

$$
\left\{\begin{array}{l}
\mu u^{\prime \prime}+A_{1}(t) u^{\prime}+B_{1}(t) u+C_{1}(t) v=-\mu \tilde{z}^{\prime \prime}(t) \\
v^{\prime}+A_{2}(t) u^{\prime}+B_{2}(t) u+C_{2}(t) v=0
\end{array}\right.
$$

with boundary conditions

$$
\begin{aligned}
& h_{1} u(t, \mu)=0 \\
& h_{2} u(t, \mu)=\beta-h_{2} \widetilde{z}(t) \\
& h_{3} v(t, \mu)=0
\end{aligned}
$$

Since in the boundary conditions (38) we did not use the condition $h_{2} \tilde{z}(t)$. Therefore in the conditions (40) $\beta-h_{2} \tilde{z}(t) \neq 0$. Problem (39), (40) is of the same type as problem (1), (2). Then by virtue of Theorem 1 we have the following estimates for $u(t, \mu), v(t, \mu)$ :

$$
\begin{gathered}
\left|u^{(j)(}(t, \mu)\right| \leq C\left(\left|\beta-h_{2} \tilde{z}(t)\right|+\mu\right)\left(1+\frac{1}{\mu^{j}} e^{-\delta \frac{t}{\mu}}\right), \\
j=0,1 \\
|v(t, \mu)| \leq C\left(\left|\beta-h_{2} \tilde{z}(t)\right|+\mu\right)\left(1+e^{-\delta \frac{t}{\mu}}\right),
\end{gathered}
$$

Hence, it follows that the solution $z(t, \mu), y(t, \mu)$ does not tend to the solution $\tilde{z}(t), \tilde{y}(t)$ of the unperturbed problem (37), (38).

Now, consider the unperturbed system

$$
\left\{\begin{array}{r}
A_{1}(t) \bar{z}^{\prime}+B_{1}(t) \bar{z}+C_{1}(t) \bar{y}=F_{1}(t), \\
\bar{y}^{\prime}+A_{2}(t) \bar{z}^{\prime}+B_{2}(t) \bar{z}+C_{2}(t) \bar{y}=F_{2}(t)
\end{array}\right.
$$

with changed boundary conditions 


$$
\begin{gathered}
h_{1} \bar{z}(t)=\alpha+\Delta_{z}, \quad h_{2} \bar{z}(t)=\beta+\Delta_{J}, \\
h_{3} \bar{y}(t)=\gamma+\Delta_{y}
\end{gathered}
$$

where $\Delta_{z}, \Delta_{y}$ and $\Delta_{J}$ are initial jumps. The problem (41), (42) is called a changed unperturbed problem.

Theorem 2. Let conditions I-IV hold. Then for the difference between of the solutions of the original problem (1), (2) and changed unperturbed problem (41), (42) the following asymptotic estimates are valid as $\mu \rightarrow 0$ :

$$
\begin{gathered}
\left|z^{(j)}(t, \mu)-\bar{z}^{j}(t)\right| \leq C\left(\left|\Delta_{J}-a_{1}(0) \Delta_{z}\right|+\right. \\
\left.+\left|\Delta_{y}+A_{2}(0) \Delta_{z}\right|+\mu\right)\left(1+\frac{1}{\mu^{j}} e^{-\delta \frac{t}{\mu}}\right), j=0,1 \\
|y(t, \mu)-\bar{y}(t)| \leq C\left(\left|\Delta_{J}-a_{1}(0) \Delta_{z}\right|+\right. \\
\left.+\left|\Delta_{y}+A_{2}(0) \Delta_{z}\right|+\mu\right)\left(1+e^{-\delta \frac{t}{\mu}}\right)
\end{gathered}
$$

where $C>0, \delta>0-$ some constants independent of $\mu$.

Proof. For the functions $u(t, \mu)=z(t, \mu)-\bar{z}(t), v(t, \mu)=y(t, \mu)-\bar{y}(t)$ we havethe system (39) with boundary conditions

$$
\begin{gathered}
h_{1} u(t, \mu)=-\Delta_{z}, \quad h_{2} u(t, \mu)=-\Delta_{J}, \\
h_{3} v(t, \mu)=-\Delta_{y}
\end{gathered}
$$

Using estimates (31) to the problem (39), (44), we have

$$
\begin{gathered}
\left|u^{(j)}(t, \mu)\right| \leq C\left(\left|\Delta_{J}-a_{1}(0) \Delta_{z}\right|+\right. \\
\left.+\left|\Delta_{y}+A_{2}(0) \Delta_{z}\right|+\mu\right)\left(1+\frac{1}{\mu^{j}} e^{-\delta \frac{t}{\mu}}\right), j=0,1 \\
|v(t, \mu)| \leq C\left(\left|\Delta_{J}-a_{1}(0) \Delta_{z}\right|+\right. \\
\left.+\left|\Delta_{y}+A_{2}(0) \Delta_{z}\right|+\mu\right)\left(1+e^{-\delta \frac{t}{\mu}}\right),
\end{gathered}
$$

This yields estimates (43). Theorem 2 is proved.

From Theorem 2 we havethat the solution of the singularly perturbed BVP (1), (2) will tend to the solution of the changed unperturbed BVP (41), (42) under the following conditions:

$$
\Delta_{J}=a_{1}(0) \Delta_{z}, \quad \Delta_{y}=-A_{2}(0) \Delta_{z}
$$

Then the boundary conditions (42) in consideration of (45) take the form

$$
\begin{gathered}
h_{1} \bar{z}(t)=\alpha+\Delta_{z}, \quad h_{2} \bar{z}(t)=\beta+a_{1}(0) \Delta_{z}, \\
h_{3} \bar{y}(t)=\gamma-A_{2}(0) \Delta_{z}
\end{gathered}
$$

where $\Delta_{z}$ - initial jump of the fast variable $z(t, \mu)$.

Thus, the solution $z(t, \mu), y(t, \mu)$ of the singularly perturbed BVP (1), (2) as $\mu \rightarrow 0$ approaches the solution $\bar{z}(t), \bar{y}(t)$ of the changed unperturbed BVP (41), (46), i.e. passages to the limit take place:

$$
\begin{gathered}
\lim _{\mu \rightarrow 0} z^{(j)}(t, \mu)=\bar{z}^{(j)}(t), \quad i=0,1, \quad 0<t \leq 1 \\
\lim _{\mu \rightarrow 0} y(t, \mu)=\bar{y}(t), \quad 0<t \leq 1 .
\end{gathered}
$$

\section{Conclusion}

In this work, asymptotic estimates of the solution of an integral BVP for a singularly perturbed system of linear ordinary differential equations are obtained. The study have shown that the solution of the original singularly perturbed integral BVP does not tend to the solution of the usual unperturbed BVP. The presence of integral terms in the boundary conditions will significantly change the corresponding unperturbed problem. The solution of the original singularly perturbed integral BVP tends to the solution of the so-called changed unperturbed BVP is proved. However, the boundary conditions have changes: an initial jump of the fast variable appears. Thus, the changed unperturbed problem is presented as a problem with an additional parameter. Note that this modification of the degenerate BVP is associated with an infinitely large value of the first-order derivatives as the small parameter tends to zero. The results obtained allow us to construct asymptotic expansions of solutions of singularly perturbed nonlinear problems.

\section{Acknowledgement}

The present work was partially supported by the Grant "Inertial neural networks with unpredictable fluctuations" (2020-2022) of the Committee of Science of the Ministry of Education and Science of the Republic of Kazakhstan (Grant No.AP08856170). 


\section{References}

1. Tihonov A.N., O zavisimosti reshenij differrencial'nyh uravnenij ot malogo parametra //Matematicheskij sbornik 22(2) (1948): 193-204 (In Russian).

2. Wasow W., Singular perturbations of boundary value problems for nonlinear differential equations of the second order// Comm. On Pure and Appl. Math. 9 (1956): 93-113.

3. Pontryagin L.S. Asimptoticheskoe povedenie reshenij system differencial'nyh uravnenij s malym parametrom pri vysshih proizvodnyh. // Izv. AN SSSR, 21(31) (1957): 605-626 (In Russian).

4. Bogoliubov, N. and Mitropolskii, Y.A.Asymptotic methods in the theory of nonlinear oscillations // Hindustan Publ. Corp., Delhi. (1961):517.

5. Vishik M.I., Lyusternik L.A. Regulyarnoe vyrozhdeniei pogranichnyj sloj dlya linejnyh differencial'nyh uravnenij s malym parametrom // UMN. 12(5) (1957): 3 - 122 (In Russian).

6. Vasil'eva A., Butuzov V. and Kalachev L. The boundary function method for singular perturbation problems// ser. SIAM Studies in Applied Mathematics. Philadeplhia: SIAM, 14 (1995):219, doi: 10.1137/1.9781611970784.

7. Lomov S. Introduction to the general theory of singular perturbations / Providence, RI: American Mathematical Society, (1992):375.

8. Imanaliev M.I. Asimptoticheskie metody $\mathrm{v}$ teorii singulyarno vozmushchennyh integrodifferencial'nyh system / Frunze: Ilim, (1972): 356. (In Russian).

9. Vishik M.I., Lyusternik L.A. O nachal'nom skachke dlya nelinejnyh differencial'nyh uravnenij, soderzhashchih malyj parametr // Doklady AN SSSR. 132(6) (1960): 1242-1245. (In Russian).

10. Kassymov K.A. Ob asimptotike resheniya zadachi Koshi s bol'shimi nachal'nymi usloviyami dlya nelinejnyh obyknovennyh differencial'nyh uravnenij, soderzhashchih malyj parametr // Uspekhi mat. nauk. 17(5) (1962): 187-188.(In Russian).

11. Kassymov K.A. Asymptotika resheniya zadachi s nashalnym skachkom dliya nelineynyx system differencialnyx uravnenii $s$ malym parametrom pri starshei proizvodnoi //DAN SSSR. 189(5) 1969: 941-944.(In Russian).

12. Kassymov K.A. Asymptotika resheniya zadachi s nashalnym skachkom dliya nelineynyx system differencialnyx uravnenii gyperbolicheskogo typa s malym parametrom pri starshei proizvodnoi //DAN SSSR, 196(2) (1971): 274-277. (In Russian).

13. Abil'daev E.A., Kassymov K.A. Asimptoticheskie ocenki reshenij singulyarno vozmushchennyh kraevyh zadach s nachal'nymi skachkami dlya linejnyh differencial'nyh uravnenij // Differencial'nye uravneniya, 28(10) (1992): 1659-1668. (In Russian).

14. Kassymov K.A., Nurgabyl D.N. Asymptotic Behavior of Solutions of Linear Singularly Perturbed General Separated Boundary-Value Problems with Initial Jump // Ukrainian Mathematical Journal. 55(11) (2003): 1777-1792.

15 Dauylbaev M.K., Mirzakulova A.E. Asymptotic behavior of solutions of singular integrodifferential equations // Journal of Discontinuity, Nonlinearity, and Complexity, 5(2) (2016): 147-154.

16. Akhmet M, Dauylbayev M.K., Mirzakulova A.E. A singularly perturbed differential equation with piecewise constant argument of generalized type // Turkish Journal of Mathematics, 42(4) (2018): 1680-1685.

17. Kassymov K.A., Tazhimuratov I.T., SheriyazdanT.T. Nerazdelennaya kraevay zadacha dlya singulyarno vozmuchennoi systemy lineynyx differrencialnyx uravnenii// Vestnik KazGU, seriamathematika, mechanica I informatika 21(2) (2000): 72-80.(In Russian).

18. Kassymov K.A., SheriyazdanT.T. Asimptoticheskie resheniya nerazdelennyx kraevyx zadach dliya singuliarno vozmuchennoi systemy lineynyx differencialnyx uravnenii // Almaty. (2001): 110.(In Russian). 